\title{
The Apathy in Dementia Methylphenidate Trial 2 (ADMET 2): study protocol for a randomized controlled trial
}

\author{
Roberta W. Scherer ${ }^{1 *}$, Lea Drye ${ }^{1}$, Jacobo Mintzer ${ }^{2}$, Krista Lanctôt ${ }^{3}$, Paul Rosenberg ${ }^{4}$, Nathan Herrmann ${ }^{3}$, \\ Prasad Padala ${ }^{5}$, Olga Brawman-Mintzer ${ }^{2}$, William Burke ${ }^{6}$, Suzanne Craft ${ }^{7}$, Alan J. Lerner ${ }^{8}$, Allan Levey ${ }^{9}$, \\ Anton Porsteinsson ${ }^{10}$, Christopher H. van Dyck ${ }^{11}$ and the ADMET 2 Research Group
}

\begin{abstract}
Background: Alzheimer's disease (AD) is characterized not only by cognitive and functional decline, but also often by the presence of neuropsychiatric symptoms. Apathy, which can be defined as a lack of motivation, is one of the most prevalent neuropsychiatric symptoms in $A D$ and typically leads to a worse quality of life and greater burden for caregivers. Treatment options for apathy in AD are limited, but studies have examined the use of the amphetamine, methylphenidate. The Apathy in Dementia Methylphenidate Trial (ADMET) found that treatment of apathy in AD with methylphenidate was associated with significant improvement in apathy in two of three outcome measures, some evidence of improvement in global cognition, and minimal adverse events. However, the trial only enrolled 60 participants who were followed for only 6 weeks. A larger, longer-lasting trial is required to confirm these promising findings.
\end{abstract}

Methods: The Apathy in Dementia Methylphenidate Trial 2 (ADMET 2) is a phase III, placebo-controlled, masked, 6-month, multi-center, randomized clinical trial targeted to enroll 200 participants with AD and apathy. Participants are randomly assigned 1:1 to $20 \mathrm{mg}$ methylphenidate per day prepared as four over-encapsulated tablets or to matching placebo. The primary outcomes include (1) the mean difference in the Neuropsychiatric Inventory Apathy subscale scores measured as change from baseline to 6 months, and (2) the odds of having a given rating or better on the modified AD Cooperative Study Clinical Global Impression of Change ratings at month 6 compared with the baseline rating. Other outcomes include change in cognition, safety, and cost-effectiveness measured at monthly follow-up visits up to 6 months.

Discussion: Given the prevalence of apathy in $\mathrm{AD}$ and its impact on both patients and caregivers, an intervention to alleviate apathy would be of great benefit to society. ADMET 2 follows on the promising results from the original ADMET to evaluate the efficacy of methylphenidate as a treatment for apathy in AD. With a larger sample size and longer follow up, ADMET 2 is poised to confirm or refute the original ADMET findings.

Trial registration: ClinicalTrials.gov, NCT02346201. Registered on 26 January 2015.

\footnotetext{
* Correspondence: rschere1@jhu.edu

'Johns Hopkins University Bloomberg School of Public Health, Baltimore, MD, USA

Full list of author information is available at the end of the article
} 


\section{Background}

Alzheimer's disease (AD) is a growing public health problem with a global burden expected to exceed 80 million cases by 2040 [1]. This disease negatively impacts patients and families both emotionally and economically [2], with societal costs at about US\$236 billion per year in the USA alone in 2016. Although cognitive and functional decline define $\mathrm{AD}$, neuropsychiatric symptoms, such as agitation, delusions, hallucinations, depression, sleep disturbance, and problem behaviors, afflict almost all patients [3]. These symptoms lead to worse quality of life, greater disability, accelerated cognitive or functional decline, greater burden on caregivers, earlier institutionalization, and accelerated mortality [2]. Apathy is one of the most prevalent neuropsychiatric symptoms in $\mathrm{AD}[4,5]$. Clinically significant apathy is defined as a loss of will and initiative, lack of interest in activities, lack of productivity, and limited affective response to positive or negative events [6] and that is present for at least 4 weeks [7]. Apathy has been reported to affect more than half of people with dementia [8] and has devastating effects on the quality of life for both patients with $\mathrm{AD}$ and their caregivers. Patients suffering from apathy experience decreased motivation, relying heavily on caregivers to initiate and oversee daily activities. Those caregivers who lack an understanding of apathy as a syndrome may misinterpret apathetic patients as insensitive and uncaring [9] and report significant levels of distress and fewer positive experiences associated with caregiving than caregivers of nonapathetic patients with AD [10, 11]. Greater caregiver distress is linked with increased service utilization and accelerated institutionalization [12], which in turn creates a significant financial burden $[13,14]$. Most notably, of all the neuropsychiatric symptoms apathy is the only symptom with high prevalence and marked persistence over the course of dementia [15]. Therefore, the management of apathy is a major priority in caring for patients with $\mathrm{AD}$ and reducing its public health burden.

There are no proven interventions to treat apathy in $\mathrm{AD}$, but the use of catecholaminergic agents for the treatment of apathy is a promising and feasible approach to repurposing available medications for this purpose. This approach is based on the understanding that motivated behaviors rely not only on the dopaminergic mesolimbic brain reward system [16] but on newly evolved prefrontal cortical circuits that degenerate in $\mathrm{AD}$, and where methylphenidate enhances both noradrenergic and dopaminergic signals to strengthen function [17]. Evidence for the use of catechoaminergic agents comes from case reports and small open-label studies in non-demented populations [18-21]. Short-acting methylphenidate has been one of the most studied catecholaminergic compounds in the elderly and presents a good safety profile [22]. It is well-tolerated during clinical use for the treatment of attention deficit hyperactivity disorder in children and young adults, the current indication approved by the Food and Drug Administration.

Data on the use of methylphenidate for the treatment of apathy in AD are sparse, but supported by case reports of methylphenidate for the treatment of apathy among adults and elders with major depression [23, 24], Parkinson's disease [25], stroke [14], and in one instance for $\mathrm{AD}$ [26], and an open-label trial of methylphenidate in vascular dementia [19]. Additional evidence is provided by two pilot randomized clinical trials. The first randomized, placebo-controlled trial of methylphenidate for the treatment of apathy in AD was a small crossover study that suggested that methylphenidate is modestly effective in most patients with AD [27-29]. The second, larger trial, the Apathy in Dementia Methylphenidate Trial (ADMET) found that methylphenidate treatment of apathy in AD was associated with significant improvement in two of three efficacy outcomes, a suggestion of an improvement in global cognition, and minimal adverse events [30,31]. These data suggest that methylphenidate might be a safe and efficacious treatment for apathy in $\mathrm{AD}$, although both randomized trials were small and had short follow up. To clarify the clinical efficacy of methylphenidate for apathy in AD more precisely, we are conducting a larger, longer trial with more robust measures: the Apathy in Dementia Methylphenidate Trial 2 (ADMET 2). ADMET 2 is a phase III, placebo-controlled, masked, 6-month, multicenter randomized clinical trial sponsored by the National Institute of Aging (NIA) involving 200 participants with AD. The trial is designed to examine the efficacy and safety of methylphenidate as treatment for clinically significant apathy in participants with $\mathrm{AD}$, where efficacy will be measured by looking at changes in apathy and cognition. ADMET 2 will enroll participants from real-world settings and examine the effects of methylphenidate on apathy, cognition, cost-utility, and safety.

\section{Methods/design}

The primary objective of ADMET 2 is to examine in a masked, placebo-controlled randomized trial the efficacy of methylphenidate for the treatment of clinically significant apathy in participants with AD. Additional objectives are to examine the cognitive effects, safety, and cost-effectiveness by assessing quality of life and economic assessment from baseline to 6 months. These assessments will be administered at baseline and at in-person follow-up visits, held monthly until 6 months after randomization. ADMET 2 is registered at ClinicalTrials.gov (NCT02346201) and is funded by the NIA, National Institutes of Health (NIH). 
The Sponsor has no role in study design, data collection or management, or writing of the final reports.

\section{Organization}

Personnel in ADMET 2 will include a chair's office $(\mathrm{CO})$, coordinating center (CC), ten clinical centers and various committees. Primary decision-making bodies are the Executive and Steering committees, while data and safety monitoring will be conducted by an independent Data and Safety Monitoring Committee (DSMC). Additional committees are those on training and certification, recruitment, quality assurance, publication and presentation, ancillary studies, and policy and protocol. The CO is responsible for coordinating study meetings, training clinical staff on cognitive and apathy assessments, and preparing recruitment materials. The CC is responsible for maintaining data integrity, managing adverse event reporting, communicating protocol modifications, and preparing all materials associated with study meetings.

Current clinical sites, responsible for recruiting, treating, and following study participants include: Emory University, Atlanta, GA, USA; Johns Hopkins School of Medicine, Baltimore, MD, USA; Roper-St. Francis Healthcare, Charleston, SC, USA; Sunnybrook Research Institute, Toronto, ON, Canada; University Hospitals-Case Medical Center, Cleveland, OH, USA; University of Arkansas, Little Rock, AR, USA; University of Rochester, Rochester, NY, USA; Wake Forest University, Winston-Salem, NC, USA; and Yale University, New Haven, CT, USA. Banner Alzheimer's Institute, Phoenix, AZ, USA discontinued active participation after 2 years. The use of diverse centers will promote representation from ethnic minority groups.

ADMET 2 aims to enroll 200 participants with clinically significant apathy from clinical centers. Participants will either be outpatients with $\mathrm{AD}$, recruited from clinical settings at the study centers or residents of nursing homes or assisted living facilities. Inclusion criteria include those related to the presence of $A D$, apathy, mild to moderate cognition, and consent. The allowable range of cognitive impairment is as broad as possible to establish dementia diagnosis, but allow sufficient cognition for quantification of cognitive and apathy symptoms. The need for treatment for apathy is determined by the study physician when a patient has symptoms that are significant enough to require medication and "routine clinical care" has not resulted in improvement. The exclusion criteria were developed primarily to address patient safety, including known contraindications to the use of methylphenidate. Specifically, individuals with conditions that are contraindicated for methylphenidate use will be excluded as will persons with clinically significant agitation, hallucinations, or delusions. Specific eligibility criteria are itemized as shown below.

\section{Eligibility criteria}

Inclusion criteria

- Possible or probable Alzheimer's disease (National Institute of Neurological and Communicative Disorders and Stroke - Alzheimer's Disease and Related Disorders Association (NINCDS-ADRDA) criteria), with Mini-Mental State Exam (MMSE) score of 10-28 inclusive

- Clinically significant apathy for at least 4 weeks for which either

- the frequency of apathy as assessed by the Neuropsychiatric Inventory (NPI) is "Very frequently", or

- the frequency of apathy as assessed by the NPI is "Frequently" or "Often" AND the severity of apathy as assessed by the NPI is "Moderate" or "Marked"'

- A medication for apathy is appropriate, in the opinion of the study physician

- Provision of informed consent for participation in the study by potential participant or surrogate (with participant assent if the potential participant is unable to provide informed consent) and caregiver

- Availability of caregiver, who spends greater than 10 hours a week with the potential participant and supervises his/her care, to accompany the participant to study visits and to participate in the study

- Sufficient fluency, of both the potential participant and caregiver, in written and spoken English to participate in study visits, physical exams, and outcome assessments

- If female, women must be postmenopausal for at least 2 years or have had a hysterectomy

Exclusion criteria

- Currently meets criteria for Major Depressive Episode, by Diagnostic Statistical Manual of Mental Disorder - IV (TR) criteria

- Clinically significant agitation/aggression for which either

- the frequency of agitation/aggression as assessed by the NPI is "Very frequently", or

- the frequency of agitation/aggression as assessed by the NPI is "Frequently" AND the severity of the agitation as assessed by the NPI is "Moderate", or "Marked"

- Clinically significant delusions for which either

- the frequency of delusions as assessed by the NPI is "Very frequently", or

- the frequency of delusions as assessed by the NPI is "Frequently" AND the severity of the delusions as assessed by the NPI is "Moderate", or "Marked"

- Clinically significant hallucinations for which either

- the frequency of hallucinations as assessed by the NPI is "Very frequently"', or

- the frequency of hallucinations as assessed by the NPI is 'Frequently' AND the severity of the hallucinations as assessed by the NPI is "Moderate", or "Marked"

- Change to AD medications within the 30 days preceding randomization, including starting, stopping, or dosage modifications

- Change in anti-depressant (except for trazodone used for sleeping difficulties as described below) use within the 30 days preceding randomization or a period of time equal to 5 half-lives of drug,

whichever period of time is longer 


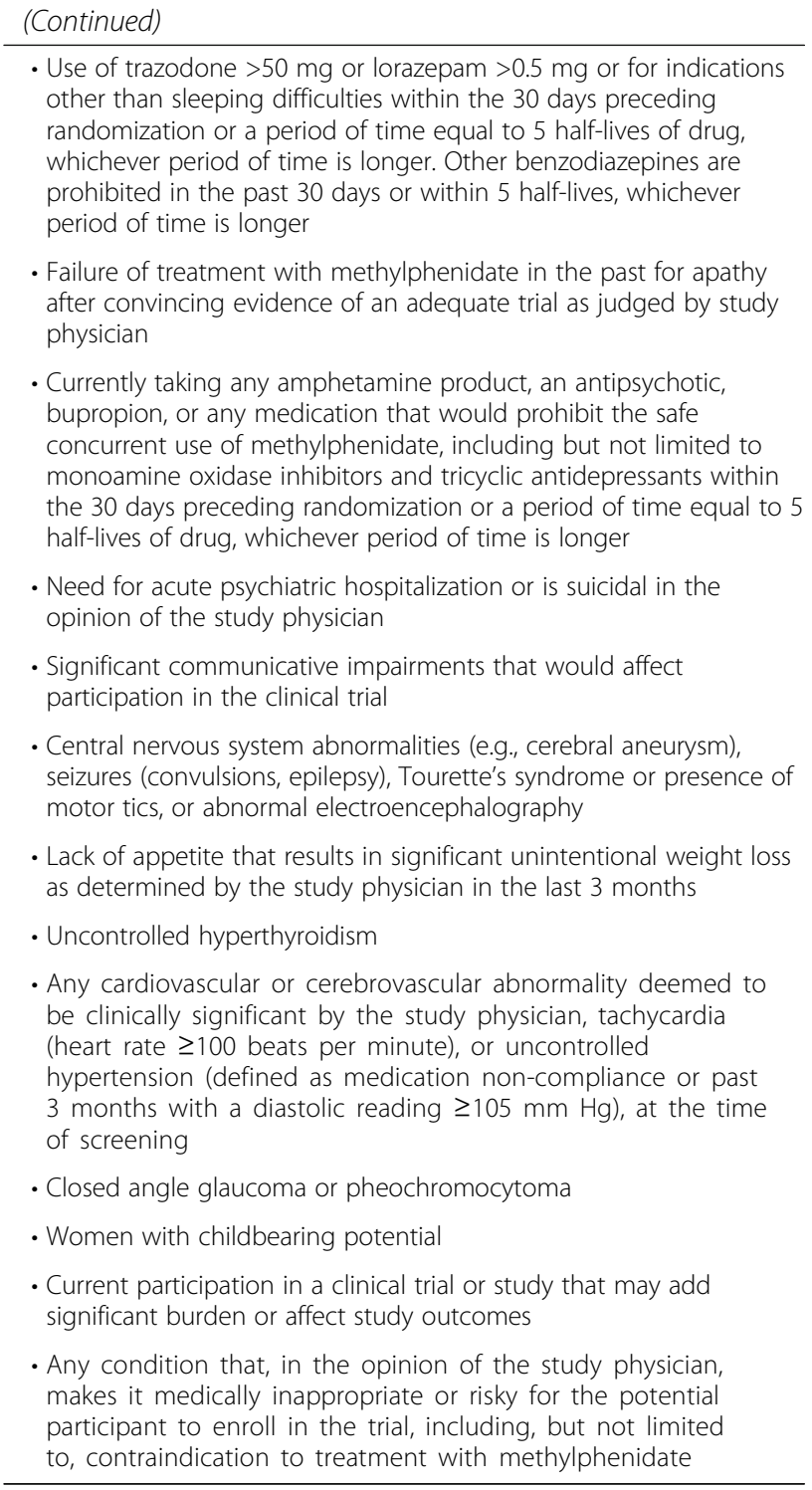

A certified study physician must evaluate the participant before randomization. The study physicians will be responsible for fully assessing whether the participant has any of the conditions listed as contraindications for the use of methylphenidate (e.g., closed angle glaucoma, hyperthyroidism, or serious unstable cardiovascular or heart rhythm). The presence of any contraindicated condition or medication will constitute an exclusion criterion for ADMET 2 . Laboratory tests for the purpose of qualifying a participant as having $\mathrm{AD}$ (e.g., brain imaging, blood and urine test, etc.) may be obtained prior to entry per current clinical standards and guidelines, but will not be required. The baseline visit may take place across more than one day, but must be completed within the 3 weeks preceding randomization.
To allow for generalizability to the usual clinical practice, ADMET 2 will allow concomitant use of a broad range of medications. The only exceptions are the use of medications that would prohibit the safe concurrent use of methylphenidate (any amphetamine product, bupropion, monoamine oxidase inhibitors, and tricycle antidepressants) or that function as dopamine receptor antagonists (antipsychotics). Changes in Alzheimer's disease medications during the study will be allowed if the clinician supervising the participant's care determines the change to be clinically required and the clinician believes that the medication will not cause or exacerbate the participant's apathy. For the treatment of sleeping difficulties, trazodone up to $50 \mathrm{mg}$ before sleep or lorazepam up to $0.5 \mathrm{mg}$ before sleep may be used nightly, or as needed. Use of other benzodiazepines or hypnotics will be prohibited.

\section{Interventions}

Participants will be assigned to one of two groups: methylphenidate plus a psychosocial intervention for the caregiver, or placebo plus psychosocial intervention for the caregiver. The psychosocial intervention, while administered to both the participant and the study caregiver, is targeted primarily at the caregiver. The target dose of methylphenidate is $20 \mathrm{mg}$ per day, provided as two over-encapsulated tablets of $5 \mathrm{mg}$ administrated orally twice a day, once in the morning and once in the afternoon. Participants assigned to methylphenidate will start on $10 \mathrm{mg}$ daily (i.e., one overencapsulated tablet of $5 \mathrm{mg}$ twice a day) for 3 days. The dose is increased to $20 \mathrm{mg}$ per day (i.e., two overencapsulated $5 \mathrm{mg}$ tablets taken twice a day) on day 4 and continues for 6 months. Participants assigned to placebo will also begin with two capsules per day and on day 4 will also begin taking four capsules a day similar to the methylphenidate group. To aid participants and caregivers, a telephone contact is expected on day 3 or immediately before the dosage increase at day 4 , and at any time a dose adjustment is required for clinical purposes. If a participant experiences unacceptable side effects on four capsules per day (20 mg methylphenidate or placebo per day), the study physician may decrease the dosage to two capsules a day $(10 \mathrm{mg}$ methylphenidate or placebo per day). Caregivers are asked to monitor and administer treatments for participants unable to do so for themselves.

For participants experiencing onset of significant agitation or delusions during the course of the trial, the study drug dose can be reduced to one capsule twice a day (10 $\mathrm{mg}$ per day in the methylphenidate group). If symptoms persist after reducing the dose the study drug can be temporarily discontinued, and can be restarted only when and if symptoms have improved and the 
participant discontinues any medication(s) used to treat the symptoms. However, study drug may only be restarted once the participant has discontinued that medication for at least 30 days or 5 half-lives, whichever is longer. If the participant requires initiation or change in an existing antidepressant (i.e., selective serotonin receptor inhibitor (SSRI)) for treatment of depression any time during the study, the study drug should be temporarily stopped until the participant is on a stable dose of the SSRI for at least 30 days or 5 half-lives, whichever is longer. Adherence to the assigned treatment will be monitored via participant and/or caregiver interview at each study visit and via pill counts. Participants and/or caregivers will be asked to return all study bottles with any unused capsules at each visit, including all empty bottles.

Participants will be monitored monthly for signs or symptoms of adverse effects. Methylphenidate is relatively short-acting with a mean half-life of 3.5 hours in adults; thus, if unexpected adverse events emerge, the investigator can simply reduce or discontinue the use of the study medication. If the unexpected adverse events disappear, the participant will re-start the study medication under close clinical supervision, including inpatient care if necessary. If the symptoms continue, it will be concluded that it is unlikely that the symptoms are related to the study medication. In that case, the participant will be treated as deemed clinically appropriate by the treating physician. The continuation or discontinuation of the drug will be decided by the center investigator in conjunction with the treating clinician based on the risk benefit consideration for each individual participant. For this reason, there will be no "rescue medication" used in this study.

Because there is no $5 \mathrm{mg}$ formulation of methylphenidate that is approved and available both in the USA and Canada, the Canadian site will use a different generic methylphenidate formulation than that used at the US sites. The two drugs have similar bioavailability based on information from the drug manufacturers. We chose this solution to allow for similar titration of methylphenidate and to allow for results that are more generalizable than using a formulation that is not approved in one of the countries.

In addition to the drug, a trained study clinician will provide a standardized psychosocial intervention modeled after the counseling strategies employed for all caregivers and participants in the previous AD trials [31-33]. The psychosocial intervention consists of a counseling session, education materials, and 24-hour availability for crises. Counseling with the caregiver (and participant if available) takes place at each study visit, lasts approximately 20-30 minutes, and includes the following elements:

- Review and adjustment of the participant and caregiver supportive care plans
- Emotional support and opportunity to ventilate feelings

- Counseling on specific caregiving skills

- Assistance with problem solving in specific issues that the caregiver brings to the sessions

- Answers to questions on the educational materials

The educational component covers AD, its clinical course, symptomatic behaviors, behavioral management of apathy, and expectations for medication treatments. This information is included in The 36-hour day [34] and Dementia care guidelines for families [2], which will be given to each caregiver. The caregiver will also be provided with 24-hour phone access to the study nurse or physician for assistance with crises that may arise after hours. The psychosocial intervention will not be administered when the participant resides in a nursing home or receives care from a paid caregiver, because the content has been developed for supporting dementia home care by family members.

\section{Recruitment}

Each site will develop their own recruitment plans tailored for potential study candidates from the local $\mathrm{AD}$ population. Recruitment activities may include chart review, telephone interviews and screens, discussion with physicians, and recruitment in the clinic waiting areas. Potential sources at each clinical center may include participants from established outpatient clinics at central or satellite locations, residents of assisted living facilities affiliated with the clinics, referrals by local physicians, or potential participants recruited from targeted advertisements in local media. The $\mathrm{CO}$ will create a prototype set of recruitment materials (i.e., brochure, poster, press release, and newspaper ad), designed to be appropriate for diverse ethnic and racial groups. Clinical centers may use these materials as part of their recruitment activities, supplementing with additional materials as needed. All recruitment materials will be approved by the local institutional review board (IRB) and be in accordance with Health Insurance Portability and Accountability Act (HIPAA) regulations to protect confidentiality.

The CC will regularly monitor recruitment by sending weekly updates to the clinic on the status of recruitment overall and of each clinic. Recruitment efforts will also be regularly reported to the Executive Committee and the Data and Safety Monitoring Committee.

\section{Randomization and masking}

The CC generated random treatment assignment schedules using a documented program in SAS 9.2 (Copyright $\odot$ 2002-2008 by SAS Institute Inc., Cary, NC, USA). The randomization schedule was designed to yield an assignment ratio of one to one for the two treatment 
groups, stratified by clinical center and using a permuted block design with random block sizes. Study participants and clinical center personnel, but not a restricted set of personnel at the CC, will be masked to treatment assignments,. The $\mathrm{CC}$ also generated a list of randomly ordered medication identifiers, which is linked to the assignment schedule. Documentation of the randomization generation processes will be retained at the $\mathrm{CC}$ and be accessible only to authorized personnel.

Treatments will be assigned using an online program accessible to the clinical centers through the web-based data system. After the entry of specified prerandomization data, and confirmation of eligibility, each enrolled participant's ID will be irrevocably linked to the next unassigned treatment for that clinical center. The clinical centers will be directed to issue a coded medication bottle containing the proper assigned treatment from among those available at the center. The data system will check to prevent randomization of the same participant ID. At each subsequent in-person follow-up visit, clinical center personnel will re-enter the participant ID to request a coded medication bottle for the next month's supply of study drug. No study drug will be issued at the 6-month follow-up visit.

Clinical centers may request an emergency unmasking from the ADMET 2 data system and must contact the CC immediately following an emergency unmasking. Optional unmasking may occur for each participant after 6 months of follow up and when all data collection is completed. The treatment assignment will be provided in a sealed envelope to the participant and/or caregiver, but not revealed to the ADMET 2 staff at the clinical centers.

\section{Outcomes}

The primary outcomes in ADMET 2 will measure the change in apathy from baseline to 6 months and include (1) mean difference in change from baseline to 6 months in the Neuropsychiatric Inventory Apathy subscale (NPI apathy) [35] scores; and (2) odds of having a given rating or better on the Modified AD Cooperative Study Clinical Global Impression of Change (mADCS-CGIC) [33] ratings at month 6. Although the original ADMET trial included the Apathy Evaluation Scale (AES) [36], we chose not to include this as a primary outcome in ADMET 2. The AES requires a notable degree of clinician judgment while interviewing the caregiver, making it difficult to implement consistently across multiple centers and multiple psychometricians. A secondary apathy outcome measure is the Dementia Apathy Interview and Rating (DAIR) [37] scale. Other secondary outcomes will include the $\mathrm{AD}$ Cooperative Study-Activities of Daily Living Scale (ADCS-ADL) [38], Dependence Scale [39], and Information on caregiver distress (NPI caregiver distress score). Cost-effectiveness will be measured using assessment of health-related quality of life (EuroQol (ED-5D-5 L)) [40], and resource utilization (Resource Utilization in Dementia Lite (RUD-lite)) [41]. A battery of cognitive tests will be assessed at baseline and at the in-person follow-up visits at 2, 4, and 6 month, including the Mini-Mental State Exam (MMSE) [42], Hopkins Verbal Learning Test Revised (HVLT-R) [43], the Digit Span: The Wechsler Adult Intelligence Scale - Revised Digit Span sub-test [44], Trail Making Tests (A and B) [45], Action Verbal Fluency Test from the Parkinson's Disease-Cognitive Rating Scale [46], Category Fluency Task-Animal Naming [47], and the Short Boston Naming Test [48]. These tests are described in detail in Table 1.

Safety will be assessed by measuring vital signs, electrolytes, electrocardiograms (ECGs), and the NPI at each in-person visit. Adverse events reports will be completed as required at each follow-up visit. These measures, chosen based on the known possible side effects of methylphenidate, will be assessed at scheduled study visits and include blood pressure, pulse, respiratory rate, electrolyte levels (sodium, potassium, chloride, bicarbonate, glucose, urea nitrogen, and creatinine), ECG, and weight. Any clinically significant change in one of these measures will be considered an adverse event. In addition, ADMET 2 has compiled a list of known side effects of methylphenidate that are monitored at scheduled visits by interview of participants and their caregivers. All serious adverse events (SAEs), as defined by the Food and Drug Administration, will be recorded with study physicians rating the severity and relatedness of the event and any associated medical care.

\footnotetext{
Known side effects of methylphenidate collected as adverse reactions in ADMET 2

Behavioral

- Aggressive behavior or hostility

- Agitation

- Anxiety, nervousness, or tension

- Depressed mood

- Distractibility

- Drowsiness

- Hyperactivity

- Impaired learning

- Impulsivity

- Insomnia

- Libido changes

Cardiovascular

- Angina

- Blood pressure changes

- Cardiac arrhythmia or serious heart rhythm abnormalities
} 


\begin{tabular}{|c|}
\hline - Palpitations \\
\hline - Peripheral vasculopathy (including Raynaud's) \\
\hline - Pulse changes \\
\hline - Tachycardia \\
\hline - Vasculitis \\
\hline Gastrointestinal \\
\hline - Abdominal pain \\
\hline - Abnormal liver function \\
\hline - Anorexia \\
\hline - Decreased appetite \\
\hline - Nausea \\
\hline - Weight loss \\
\hline Hematologic \\
\hline - Anemia \\
\hline - Thrombocytopenic purpura \\
\hline Musculoskeletal \\
\hline - Arthralgia (joint pain) \\
\hline - Dyskinesia (abnormal movement) \\
\hline - Muscle stiffness or aching, muscle tenderness \\
\hline - Rhabdomyolysis \\
\hline Neural \\
\hline - Blurry vision or eyesight changes \\
\hline - Dizziness \\
\hline - Dry mouth \\
\hline - Headache \\
\hline - Numbness of fingers, toes, nose, ears, lips \\
\hline - Tics (motor or verbal) \\
\hline Other \\
\hline - Fever \\
\hline - Hair loss \\
\hline - Priapism \\
\hline - Serotonin Syndrome \\
\hline - Skin rash, redness, or inflammation \\
\hline - Urine color change or decreased output \\
\hline - Urticaria \\
\hline
\end{tabular}

\section{Study visits}

Follow up will include both scheduled and unscheduled visits and contacts. Scheduled follow up will include inperson visits scheduled at monthly intervals after randomization, telephone contacts for data collection (days 15, 45, and 75 after randomization), and telephone contact for dose adjustments (day 3 and as needed). Target dates for follow-up visits will be calculated from the date of randomization. At all scheduled in-person visits, study staff will review study procedures to verify ongoing consent, the interim medical history and current medications. Assessments completed at each in-person visit will include $\mathrm{AD}$ and safety assessments. At months 2, 4, and 6, study staff will also collect functional assessments and perform the cognitive battery, and at months 3 and 6 , study staff will perform cost-utility assessments. Data collected at the telephone contacts will include interim medical history, compliance, and adverse events. The specific data instruments administered at each scheduled contact are shown in Table 2.

\section{Sample size}

Power calculations were conducted for the two primary outcomes: (1) mean difference in change from baseline to 6 months in the NPI apathy subscale scores; (2) odds of having a given rating or better on the mADCS-CGIC ratings at month 6 . Both calculations assumed a type I error rate of 0.025 to preserve an overall type I error rate of 0.05 over both primary comparisons.

The power and sample size for the NPI apathy outcome were determined with standard two-sample methods for comparing means using SAS 9.2 (Copyright (c) 2002-2008 by SAS Institute Inc., Cary, NC, USA). The sample size calculations assumed the difference in NPI apathy change scores (1.8 points) and standard deviation (3.2) of the change scores observed in the original ADMET study [31]. The difference in NPI apathy is similar to the difference between mean change in "moderate improvement" and "minimal improvement" or "no change" observed in the ADMET trial. A sample size of 200 participants will ensure greater than $90 \%$ power to detect a difference of 1.8 points in change on the NPI apathy scale even if $15 \%$ of the participants are lost before the 6-month visit.

The power and sample size for the mADCS-CGIC outcome were determined using the method of Whitehead [49] for proportional odds logistic regression implemented by the "popower" in the Hmisc package in $\mathrm{R}$ ( $\mathrm{R}$ version 2.1.3.1, Copyright (c) by The $\mathrm{R}$ Foundation for Statistical Computing).

We assumed the overall proportions of ratings in each category from ADMET: $3.5 \%$ with marked improvement; $8.8 \%$ with moderate improvement; $29.8 \%$ with minimal improvement; $54.4 \%$ with no change; $3.5 \%$ with minimal worsening; and $0 \%$ with moderate or marked worsening. Assuming an odds ratio for better ratings in methylphenidate of 2.75 (about 25\% smaller than the odds ratio observed in ADMET) and 200 participants, the study will have $90 \%$ power to detect a difference between treatment groups with $10 \%$ losses to follow up and greater than $85 \%$ power with $15 \%$ losses.

\section{Data collection and management}

ADMET 2 staff will collect study data on paper data collection forms and enter data online using the 
Table 1 Description of data collection instruments used in ADMET 2

\begin{tabular}{|c|c|c|}
\hline Instrument & Domains measured & Scoring \\
\hline \multicolumn{3}{|l|}{ A. Apathy } \\
\hline $\begin{array}{l}\text { Modified AD } \\
\text { Cooperative } \\
\text { Study-Clinical Global } \\
\text { Impression of Change } \\
\text { (mADCS-CGIC) }\end{array}$ & Change in apathy & $\begin{array}{l}\text { Seven-point scale where } 1 \text { is "very much } \\
\text { improved" and } 7 \text { is "very much worse"; } \\
\text { a rating of } 4 \text { being "no change" }\end{array}$ \\
\hline $\begin{array}{l}\text { Neuropsychiatric } \\
\text { Inventory (NPI) }\end{array}$ & $\begin{array}{l}\text { Frequency and severity of neuropsychiatric } \\
\text { symptom (apathy, agitation, delusions, } \\
\text { hallucinations, depression, euphoria, aberrant } \\
\text { motor behavior, irritability, disinhibition, } \\
\text { anxiety, sleeping, and eating disorders) }\end{array}$ & $\begin{array}{l}\text { Scores determined by multiplying frequency } \\
\text { (scored from } 1 \text { to } 4 \text { ) and severity (scored from } \\
1 \text { to } 3 \text { ) with caregiver distress (scored } 1 \text { to } 5 \text { ) } \\
\text { and adding caregiver distress (scored } 1 \text { to 5); } \\
\text { total score is sum of the score for each of the } \\
12 \text { (range is from } 0 \text { to } 144 \text { ). Higher scores } \\
\text { indicate greater frequency and severity of } \\
\text { symptoms }\end{array}$ \\
\hline $\begin{array}{l}\text { Dementia Apathy } \\
\text { Interview and Rating } \\
\text { (DAIR) }\end{array}$ & $\begin{array}{l}\text { Used to discriminate between apathy from } \\
\text { lack of interest due to personality traits and } \\
\text { evaluates change in motivation, engagement, } \\
\text { and emotional response since disease onset }\end{array}$ & $\begin{array}{l}\text { Each of } 16 \text { items scored from } 0 \text { to } 3 \text {, with } \\
\text { apathy score a sum of all items administered, } \\
\text { divided by the number of items completed. } \\
\text { Total scores range from } 0 \text { to } 3 \text {, with higher } \\
\text { scores representing more apathy }\end{array}$ \\
\hline
\end{tabular}

Administration

Based on interview with caregiver by independent skilled, and experienced clinician

Interview with caregiver by clinician

Interview with caregiver by clinician

Scored from 0 to 15 , with higher scores indicating an increased level of dependence

Scale discriminates between the stages of severity of $A D$, from very mild to severely impaired

Each domain graded from level 1 (no problems) to level 5 (extreme problems) usual activities, pain/discomfort, and anxiety/depression

Resource Utilization in Assesses resource utilization and includes Dementia-Lite (RUD-Lite) questions on accommodation, informal care, community care, and hospitalizations

D. Cognition

Mini-Mental State Exam (MMSE)

Hopkins Verbal Learning Test Revised (HVLT-R)

Digit Span: the Wechsler Adult Intelligence ScaleRevised Digit Span sub-test

Trail Making Tests (A and B)

Action Verbal Fluency Test from the Parkinson's Disease-

Cognitive Rating Scale

Category Fluency Task-Animal Naming

\section{General cognition}

Cognition: verbal learning, recognition, and delayed recall

Cognition: auditory attention and working memory

Cognition: attention, executive function, and visuo-motor tracking

Cognition: executive function, working memory, and information processing speed

Cognition: executive function, working memory, set shifting, and executive control

Short Boston Naming Cognition: expressive language Test
Presence or absence of resource use

Scored from 0 to 30, with higher scores indicating higher cognitive functioning.

Scored from 0 to 12 , with higher scores indicating better performance

Separate scores are obtained for spans read forwards and backward from 0 to 9 for the number of digits correctly identified and for the longest span that is recalled.

Time taken to complete the test with shorter time indicating higher cognitive functioning

Score is the total number of unique verbs, with higher counts indicating less cognitive

Scores are the number of animals verbalized, with higher counts indicating less cognitive impairment

The minimum score is 0 and the maximum score is 15 . Higher scores indicate better control of expressive language impairment
Based on interview with caregiver by clinician

Based on structured interview of caregiver by clinician

Interview of participant and/or caregiver by clinician

Interview of participant and/or caregiver by clinician

Administered by trained interviewer to participant

Administered by trained interviewer to participant

Administered by trained interviewer to participant

Administered by trained interviewer to participant

Administered by trained interviewer to participant

Administered by trained interviewer to participant

Administered by trained interviewer to participant 
Table 2 Data collection by visit

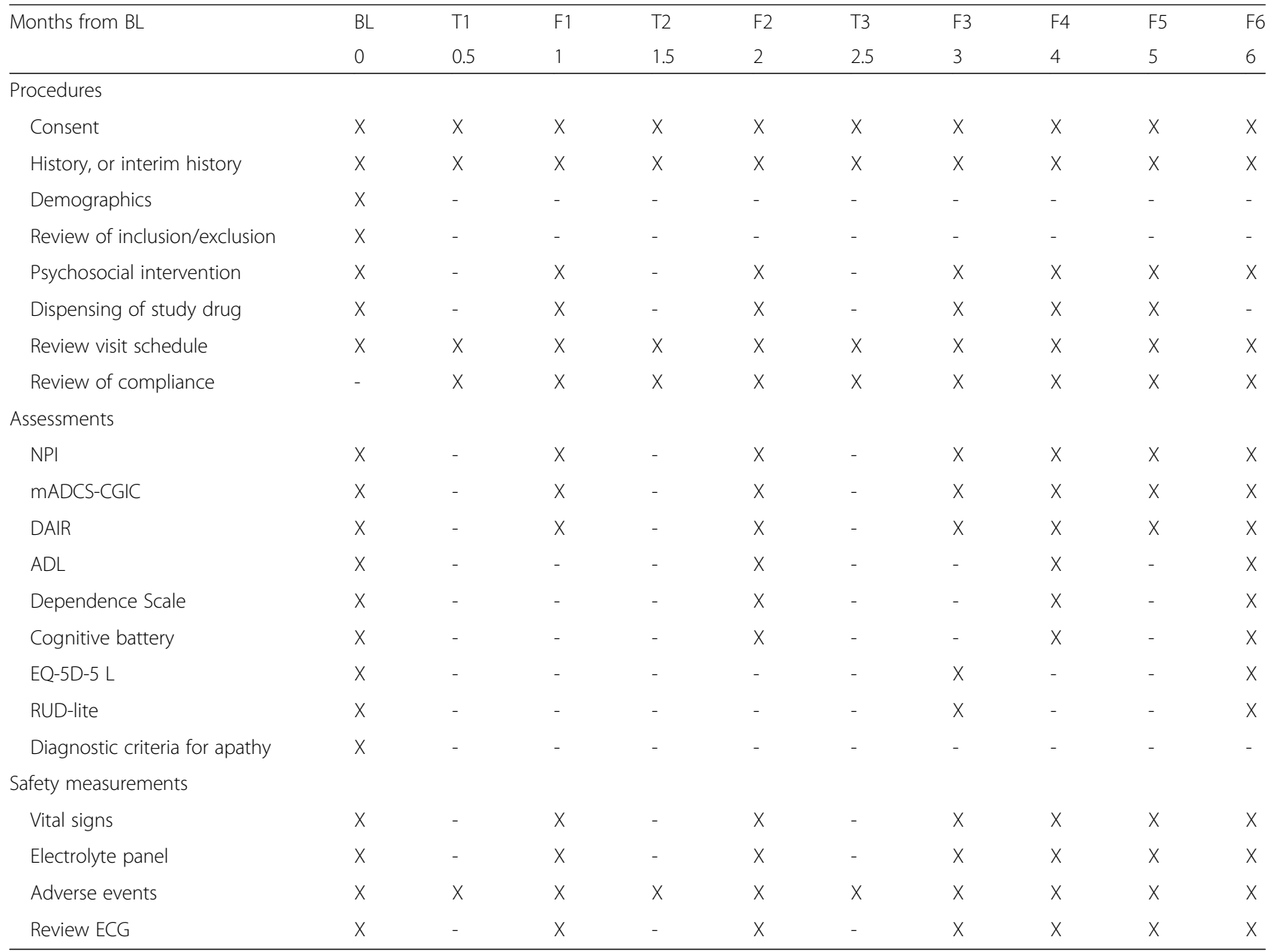

$B L$ baseline visit, $F$ scheduled follow-up visits, $T$ scheduled telephone contact, NPI Neuropsychiatric Inventory, mADCS-CGIC modified AD Cooperative Study Global Impression of Change, DAIR Dementia Apathy Interview and Rating, $A D L$ Activities of Daily Living, EQ-5D-5 L Euro Quality of Life, $R U D$-lite Resource Utilization in Dementia-Lite, ECG electrocardiogram

ADMET 2 website (http://admet.org/Public/ADMET Public.html). Names, addresses, and other such personal data of participants and caregivers will not be entered and will not be part of the central database. Data collected from study evaluations and interviews will be identified only by study ID codes, which include the pre-specified participant ID and a four-letter code assigned at eligibility evaluation. Caregivers will also be identified by a unique ID code. The study will employ double data entry and validation checks against criteria specified in the data dictionary, including checks for consistency with other responses, and completeness. Data errors and inconsistencies will be flagged during the data entry process. Security measures will include password protection on a need to know basis for ADMET 2 staff. The database will be backed up daily and weekly on high-capacity cassette tapes. Monthly backups will be stored to $\mathrm{CD}$ media.

\section{Statistical methods}

All primary analyses will be based on the "intention to treat" principle. Initial analyses will be descriptive in nature, using means, standard deviations, and proportions to describe baseline characteristics of the sample for all participants combined, and by intervention group. We will assess comparability of all baseline factors among the randomly assigned groups using appropriate statistical methods. Pearson correlation coefficients (or Spearman rank correlation) will be calculated to assess the strength of the associations between covariates and outcome measures; those with potential for confounding will be considered for inclusion in secondary analyses involving regression models. As required by NIH policy, we will conduct valid subgroup analyses of the primary and adverse event outcomes by gender and race/ethnicity to determine if there are possible differences (i.e., interactions) in treatment effects. 
The primary outcome of difference in change from baseline to six months in the NPI apathy subscale scores will be analyzed initially by a crude comparison of the difference in mean change scores from baseline to month 6 using the $t$ test. Longitudinal analyses of NPI apathy scores to compare treatment groups over time will use a saturated means model (including indicators for each visit and each visit-by-treatment interaction) adjusting for randomization stratification (clinic) by creating a linear mixed-effects model with random intercept for each participant to account for multiple measurements over time. The primary comparison is difference in mean change from baseline to month 6 . A transformation of the outcome data will be used, if needed, to meet model assumptions. We will conduct planned subgroup analyses to see if treatment effects differ for (1) The US sites versus the Canadian site to account for the different generic methylphenidate used at these sites, (2) participants who meet the proposed diagnostic criteria for apathy at baseline versus those who do not meet criteria, and (3) apathy severity at baseline. Planned sensitivity analyses include comparison of the rate of change in NPI apathy scores over 6 months using target and actual visit times, using a subset of adherent participants and comparison of the proportion of participants with greater than 30\% reduction in NPI apathy scores at 6 months.

The primary outcome of ratio of the odds of being at or better than a given category on the mADCS-CGIC at month 6 will be analyzed using proportional odds logistic regression to compare the mADCS-CGIC ratings of change (ranging from "marked worsening" to "marked improvement") at month 6 between the treatment groups. The categorical outcome on the mADCS-CGIC of each participant at month 6 measures each participant's overall apathy at the endpoint relative to that at the baseline visit on a 7-point scale $(1=$ marked improvement, $2=$ moderate improvement, $3=$ minimal improvement, $4=$ no change, $5=$ minimal worsening, $6=$ moderate worsening and $7=$ marked worsening) and will be compared by assigned randomization group. If the data do not meet the proportional odds assumption, the Wilcoxon rank-sum test will be used to compare the mADCS-CGIC ratings. We will perform the same subgroup analyses described for the change in NPI apathy score.

Safety outcomes will be analyzed by assessing the adverse events data collected at each in-person visit, including those identified using systematic, close-ended questions for known or expected side effects of methylphenidate and open-ended questions for unexpected side effects, and abnormal results of electrolyte or ECG results. The proportion of participants experiencing adverse events and SAEs will be compared between treatment groups using logistic regression or Fisher's exact test, controlling for baseline imbalances if necessary. To assess the superiority of methylphenidate compared with placebo for effects on cognitive and functional outcomes, we will look at longitudinal change in the DAIR, ADCS-ADL, Dependence Scale, and cognitive sphere scores from baseline to month 6 using a mixed-effects model as described above. For the pharmaco-economic analyses, costs (direct, indirect, and caregiver as reported in the RUD-Lite) will be summed for each treatment arm and compared with nonparametric tests, as cost data are typically skewed. We will conduct a cost-utility analysis by comparing total costs in each treatment arm with summation of utilities derived from the EQ-5D-5 L assessment to generate a cost per quality-adjusted life year (QALY). This will provide an important assessment of the cost-effectiveness of the intervention as we anticipate that treating with methylphenidate versus placebo will be economically attractive (i.e., potentially cost-saving if improved apathy is associated with lower overall costs and superior levels of functioning and quality of life).

For study participants with missing outcomes, multiple imputation will be considered, and analyses will follow the plans described above and the combination rules for multiple imputation. To investigate sensitivity to missing values, study participants with and without missing values will be compared by background covariates, and any observed differences will be adjusted for in the analyses.

\section{Quality control and performance monitoring}

All ADMET 2 staff will be certified prior to performing trial activities to document a minimum level of competency to perform the functions of that role. In addition, each person requesting certification must sign a personal assurance of integrity in the data collection process, have completed a human subject/ethics training course within the last 5 years, and sign and complete the International Committee of Medical Journal Editors (ICMJE) Uniform Disclosure Form for Potential Conflicts of Interest. Persons completing the cognitive battery will be required to have administered at least seven of each type of assessment before being certified to complete that assessment. Study investigators will also be required to complete a clinical center certification form documenting sufficient space, facilities, and personnel and the requisite ethical approval to conduct the study.

Centers will be monitored centrally on a regular basis for rate of enrollment, protocol deviations, number and proportion of missed visits and losses to follow up, completeness of data, percentage of data items requiring edit queries, and percentage of discrepancies found in audited data items. In addition, site visits will be made to each of the clinical centers early in the course of recruitment and at other points in time as needed or desired for quality assurance purposes. Periodic audits of 
subsets of the database will also be conducted, both through visits to the centers and through a remote auditing procedure. At on-site visits, participant data will be chosen for verification from source documentation. For the remote auditing procedure, the $\mathrm{CC}$ will periodically review participant form sets.

\section{Data monitoring}

The ADMET 2 DSMC committee will review accumulating data to monitor participant safety and evaluate the efficacy of methylphenidate for the treatment of apathy. They will provide a summary report of recommendations to the study chair and the CC following each meeting, and to the Steering Committee and the NIA in their capacity as an advisory committee. The DSMC will have three members, each of whom will have a background in geriatrics and psychopharmacological research related to $\mathrm{AD}$, and an ex officio NIA representative. Formal analysis of the primary outcome data will be conducted and presented to the DSMC when 50\% (at least 100 participants) of the expected participants have been enrolled in the study. The DSMC will not have any formal stopping rule, but will regularly review and evaluate accumulating safety data and may recommend termination of the trial if the risks become unacceptable.

Adverse events and SAEs will be defined as used in the DIADS-2 study [32]. Center investigators will be responsible for appropriate medical care of participants during the study, in connection with study procedures, and for monitoring the safety of participants. A medical monitor designated by the study chair will provide consultation to all centers on medical monitoring. The medical monitor and the $\mathrm{CC}$ will be notified using a special SAE report form within one working day by the investigative center via email and telephone if any of the following events occur: death, hospitalization or prolongation of hospitalization, lifethreatening events or events that involve a persistent or significant disability or incapacity, or an unexpected event. Data collected on SAEs will include the treatment provided, outcome, and presumed relationship to study drug and will be updated as new information becomes available; a narrative description also will be provided. CC personnel review the data, and following any necessary clarification, forward the report to all investigators for submission to IRBs and to the DSMC as part of their safety review. Other adverse events will include monitoring for clinically significant changes from baseline for the following: vital signs, weight loss greater than or equal to 7\%, MMSE score; NPI score, specifically for an increase in neuropsychiatric symptoms other than apathy (e.g., hallucinations, delusion, etc., and abnormal electrolytes or ECG).

\section{Ethics}

ADMET 2 will be conducted according to the ethical principles of the Declaration of Helsinki. This study received ethical approval from the IRBs of Johns Hopkins Bloomberg School of Public Health (for the CC and CO), and from IRBs of each clinical site. Both participant and caregiver (if required by the local IRB) will be required to provide consent to participate in ADMET 2. Capacity of the potential study participant to give consent will be assessed in clinical interviews by investigators trained in obtaining consent from decisionally impaired persons. Caregivers will provide consent for potential participants who are unable to provide consent; the participant will be asked to provide assent. If potential participants are able to provide informed consent, they will be asked to do so and their caregiver will co-sign the consent form as a witness. The process of obtaining consent and assent will be documented in every case. ADMET 2 clinical staff will continue to obtain assent at each subsequent visit or during implementation of study procedures to assure continuing informed consent on the part of the proxy, to maintain assent by the participants, and to assess capacity. Only individuals who can provide informed consent for themselves can be caregivers. Each caregiver will be asked to provide informed consent for participation as informant and also to provide data on themselves as caregivers in the course of the study. Caregiver informed consent will be required unless otherwise stated by the center's local IRB.

\section{Dissemination policy}

Authorship for all ADMET 2 manuscripts describing the main findings (i.e., comparisons of treatment groups) will follow hybrid corporate or group authorship format, naming "The ADMET 2 Research Group" as author, with individual investigators and clinical center staff acknowledged. Other manuscripts may have conventional authorship. The primary results will be published before presentation of the results at any conference. Decisions on timing, content, and conclusions of publications rest with the ADMET 2 Steering Committee, although some of these decisions will be delegated to a Publication Committee. No comparison data will be available prior to completion of the study and no clinic may publish data obtained from their clinic independently. Writing committees for ADMET 2 papers will include at least one representative from the study chair's office, one from the $\mathrm{CC}$, and other study group members based on interest and expertise. All ADMET 2 manuscripts will be submitted to journals complying with the NIH Public Access Policy, and all publications will be archived in PubMed Central as required by this policy. Presentations at conferences describing or presenting ADMET 2 results follow the same guidelines and require clearance by the Steering Committee. 
Public access to protocol and data

The CC will facilitate data sharing in accordance with the NIH Data Sharing Policy. Study data will be provided to all ADMET 2 investigators after data collection is complete. ADMET 2 data will become available to outside investigators at the conclusion of the trial and following publication of the main study findings as a limited use dataset with documentation. Study participants are informed about data sharing with external investigators in the consent forms. All outside investigators will be asked to sign a data use agreement to protect study participant confidentiality.

\section{Discussion}

This report described ADMET 2, a randomized trial comparing $20 \mathrm{mg}$ of methylphenidate with placebo for the treatment of apathy, one of the most prevalent neuropsychiatric symptoms of AD. ADMET 2 is planned as a larger, longer study following ADMET, a randomized trial in which methylphenidate was shown to reduce apathy in the Apathy Evaluation Scale and the mADCSCGIC, but not in the NPI. In ADMET, methylphenidate was administered only for 6 weeks and had a study population of 60 participants. In contrast, ADMET 2 plans to enroll 200 participants and follow them for 6 months. ADMET 2 is testing the efficacy of methylphenidate in reducing apathy as measured by three different instruments: mADCS-CGIC, NPI and DAIR. In addition, because ADMET study findings suggested the possibility of an improvement in cognition, ADMET 2 will administer a battery of cognitive tests aimed at distinguishing whether the apparent improvement was in fact an increase in cognition or simply an increase in attention during cognitive testing. Given the prevalence of neuropsychiatric

\begin{tabular}{|c|c|c|c|c|c|c|c|c|c|c|c|c|}
\hline \multirow[b]{2}{*}{ TIMEPOINT** } & \multirow{2}{*}{$\begin{array}{c}\begin{array}{c}\text { Enroll- } \\
\text { ment }\end{array} \\
0\end{array}$} & \multirow{2}{*}{$\begin{array}{c}\begin{array}{c}\text { Alloca- } \\
\text { tion }\end{array} \\
0\end{array}$} & \multicolumn{9}{|c|}{ Post-allocation (months) } & \multirow{2}{*}{$\begin{array}{c}\begin{array}{c}\text { Close- } \\
\text { out }\end{array} \\
6\end{array}$} \\
\hline & & & 0.5 & 1 & 1.5 & 2 & 2.5 & 3 & 4 & 5 & 6 & \\
\hline \multicolumn{13}{|l|}{ ENROLLMENT: } \\
\hline \multirow{2}{*}{$\begin{array}{l}\text { Eligibility screen } \\
\text { Informed consent }\end{array}$} & $\mathrm{X}$ & & & & & & & & & & & \\
\hline & $x$ & & & & & & & & & & & \\
\hline Allocation & & $\mathrm{X}$ & & & & & & & & & & \\
\hline \multicolumn{13}{|l|}{ INTERVENTIONS: } \\
\hline \multicolumn{13}{|l|}{$\begin{array}{r}\text { Methylphenidate and } \\
\text { psychosocial } \\
\text { intervention }\end{array}$} \\
\hline \\
\hline \multicolumn{13}{|l|}{ ASSESSMENTS: } \\
\hline$N P I$ & $\mathrm{X}$ & & & $x$ & & $x$ & & $x$ & $x$ & $x$ & $x$ & \\
\hline mADCS-CGIC & $\mathrm{X}$ & & & $\mathrm{x}$ & & $\mathrm{x}$ & & $\mathrm{X}$ & $\mathrm{x}$ & $x$ & $x$ & \\
\hline \multirow{3}{*}{$\begin{array}{r}\text { DAIR } \\
A D L \\
\text { Dependence Scale }\end{array}$} & $x$ & & & $x$ & & $\mathrm{X}$ & & $\mathrm{x}$ & $\mathrm{x}$ & $x$ & $x$ & \\
\hline & $\mathrm{X}$ & & & & & $\mathrm{X}$ & & & $\mathrm{x}$ & & $x$ & \\
\hline & $\mathrm{X}$ & & & & & $\mathrm{x}$ & & & $\mathrm{x}$ & & $x$ & \\
\hline \multirow{2}{*}{$\begin{array}{r}\text { Cognitive battery } \\
E Q-5 D-5 L\end{array}$} & $\mathrm{X}$ & & & & & $\mathrm{x}$ & & & $\mathrm{x}$ & & $x$ & \\
\hline & $\mathrm{X}$ & & & & & & & $\mathrm{X}$ & & & $\mathrm{X}$ & \\
\hline \multirow{2}{*}{$\begin{array}{r}\text { RUD Lite } \\
\text { Diagnostic Criteria for } \\
\text { Apathy }\end{array}$} & $\mathrm{X}$ & & & & & & & $\mathrm{X}$ & & & $x$ & \\
\hline & $x$ & & & & & & & & & & & \\
\hline \multirow{2}{*}{$\begin{array}{c}\text { Vital signs } \\
\text { Electrolyte panel }\end{array}$} & $\mathrm{x}$ & & & $\mathrm{X}$ & & $x$ & & $\mathrm{x}$ & $\mathrm{x}$ & $x$ & $x$ & \\
\hline & $\mathrm{X}$ & & & $\mathrm{X}$ & & $x$ & & $x$ & $x$ & $\mathrm{x}$ & $X$ & \\
\hline Electrocardiogram & $x$ & & & $x$ & & $x$ & & $x$ & $x$ & $x$ & $x$ & \\
\hline \multirow{2}{*}{$\begin{array}{r}\text { Adverse events } \\
\text { Unmasking letter for } \\
\text { participant and } \\
\text { caregiver }\end{array}$} & $\mathrm{x}$ & & $\mathrm{X}$ & $x$ & $x$ & $x$ & & $x$ & $x$ & $x$ & $x$ & \\
\hline & $\mathrm{X}$ & & & & & & & & & & & $\mathrm{x}$ \\
\hline
\end{tabular}

Fig. 1 Standard protocol items: recommendation for interventional trials (SPIRIT) figure for the Apathy in Dementia Methylphenidate Trial 2. The cognitive battery includes the following assessments: Mini-Mental State Exam, Hopkins Verbal Learning Test - Revised, Wechsler Adult Intelligence Scale - Revised Digit Span sub-test, Trail Making Tests (A and B), Action Verbal Fluency Test from the Parkinson's Disease-Cognitive Rating Scale, Category Fluency Task-Animal Naming, and the Short Boston Naming Test. Abbreviations: NPI Neuropsychiatric Inventory Apathy, mADCS-CG/C modified AD Cooperative Study Clinical Global Impression of Change, DAIR Dementia Apathy Interview and Rating, ADL Cooperative Study-Activities of Daily Living Scale, EQ-5D-5 L EuroQol 5D-5 L, RUD-Lite Resource Utilization in Dementia-Lite 
symptoms and especially apathy in $\mathrm{AD}$ and its impact on both patients and caregivers, an intervention to alleviate apathy would be of great benefit to society.

\section{Trial status}

This report describes the protocol, version 1.3, 02 May 2017 and describes the ADMET 2 protocol and adheres to the standard protocol items: recommendation for interventional trials (SPIRIT) reporting guidelines with attached checklist and figure (see Fig. 1).

ADMET 2 is currently recruiting study participants. The first study participant was randomized on January 2016. As of 1 October 2017, 77 of 200 study participants have been enrolled. The targeted end date for recruitment is June 2018.

\section{Abbreviations}

AD: Alzheimer's disease; ADCS-ADL: Cooperative Study-Activities of Daily Living Scale; ADCS-mCGIC: modified Cooperative Study Clinical Global Impression of Change; ADMET: Apathy in Dementia Methylphenidate Trial; ADMET 2: Apathy in Dementia Methylphenidate Trial 2; CC: Coordinating center; CO: Chair's office; DAIR: Dementia Apathy Interview and Rating; DSMC: Data and Safety Monitoring Committee; ECG: Electrocardiogram; HIPAA: Health Insurance Portability and Accountability Act; HVLT-R: Hopkins Verbal Learning Test - Revised; ICJME: International Committee of Medical Journal Editors; IRB: institutional review board; MMSE: Mini-Mental State Exam; NIA: National Institute of Aging; NIH: National Institutes of Health; NPI: Neuropsychiatric Inventory Apathy; RUD-Lite: Resource Utilization in Dementia-Lite; SAE: Serious adverse event

\section{Acknowledgements}

The ADMET 2 Research Group includes the following: Study Chair's Office: Jacobo Mintzer, Study Chair; David Clark, Medical Monitor; Debra Battjes-Siler, Stan Smith, Courtney O'Neil, Nicole Stocking, Office Managers. Coordinating Center: Roberta W. Scherer, Center Director; Lea Drye, Deputy Director and Biostatistician (2015-2016); Jamie Perin, Biostatistician (2016-present); David Shade, Data System Architect; Jennifer Jones, Lead Project Coordinator; Stephanie Holland, Project Coordinator; Alicia Wentz, Statistical Analyst; Shumon Chattopadhyay, Lead Data System Analyst; Bethany Grove, Stephanie Herrera, Kristen Kaiser, Andie Lears, Data System Analysts; April Broadnax, Aisha Mohammed, Research Assistants. National Institute of Aging: Laurie Ryan, Alvin McKelvy (2017-present), Cerise Elliott (2015-2017), Project Officers, Data and Safety Monitoring Committee: Kenneth Rockwood, M.D.; Steve Edland, Ph.D.; Raymond Scott Turner, Ph.D. Clinical Centers - University of Arkansas: Prasad Padala, Center Director; Debbie Hodges, Nicole Jackson (2015-2017), Lead Coordinators; Kalpana Padala, Study Physician. Banner Alzheimer's Institute, Phoenix, AZ (2015-2017): William Burke, Center Director; Dawn Batchuluun, Lead Coordinator; Anna Burke, Study Physician; Michele Grigaitis-Reyes, Marjoire DiLise-Russo, Shelia Vadovicky, Study Clinicians; Susan Favaro, Mary Lou Hernandez, Psychosocial Intervention Administrators; Lynn Autry, Nicole Hoffmann, Cognitive Assessment Raters; Rebecca Sanback, Lazaro Martinez Lujan, Coordinators; Elena Young, Data System Operator. University Hospitals - Case Medical Center: Alan Lerner, Center Director; Susie Sami, Lead Coordinator; Marianne Sanders, Study Clinician; Parianne Fatica, Maria Gross, Cognitive Assessment Raters; Ethan Gore (2015-2016), Supriya Mahajan, Study Physicians. Emory University: Allan Levey, Center Director; James Lah, Co-Investigator; Tamara Attis, Lead Coordinator; Janet Cellar, Chad Hales, Study Physicians; Margaret Walker, Study Clinician; Susan Peterson-Hazan, Psychosocial Intervention Administrator; Erin Bolles, Coordinator; Erin Carter, Cognitive Assessment Rater. Johns Hopkins University: Paul Rosenberg, Center Director; Chris Marano, Study Physician; Jasmine Dixon, Lead Coordinator; Sarah Lawrence, Cognitive Assessment Rater; Meghan Schulz, Psychosocial Intervention Administrator; Haroon Burhanullah, Study Physician. University of Rochester: Anton Porsteinsson, Center Director; Susan Salem-Spencer, Lead Coordinator; Kim Martin, Audrey Rice, Study Clinicians; Nancy Kowalski, Coordinator; Michelle Cervello, Melanie Keltz, Kaitlyn Lane, Asa Widman, Cognitive Assessment Raters.
Roper-St. Francis Healthcare: Olga Brawman-Mintzer, Center Director; Jacobo Mintzer, Co-Investigator; Anthony Awkar, Lead Coordinator; Abigail O'Connell, Psychosocial Intervention Administrator; Arthur Williams, Cognitive Assessment Rater; Sheila Howland, Alex Hails (2016), Study Clinicians; Dennis Orwat, Study Physician (2015-2016). Sunnybrook Research Institute: Krista Lanctôt, Nathan Herrmann, Center Directors; Abby Li, Lead Coordinator; Damien Gallagher, Study Physician; Adam Dinoff, Danielle Vieira, Cognitive Assessment Raters; Jennifer Bray, Psychosocial Intervention Administrator; Eleenor Abraham, Coordinator. Wake Forest University: Suzanne Craft, Center Director; Alecia Jenkins, Lead Coordinator; Deborah Dahl, Samantha Rogers, Bonnie Sachs, Study Clinicians; Emily Mann, Edward Shaw, Kaycee Sink, Study Physicians; Erin Caulder, Magie Jefferson (2015-2016), Cognitive Assessment Raters; Camilla Martin, Psychosocial Intervention Administrator; Kelsey Shore, Coordinator. Yale University: Christopher H. van Dyck, Center Director; Emily Kemp, Co-Investigator; Hannah Michalak, Lead Coordinator; Melinda Becker, Erika Pugh, Tyler Godek, Cognitive Assessment Raters; Martha MacAvoy, Study Clinician; Susan Good, Psychosocial Intervention Administrator; Megan Ebner, Julia McDonald, Srinath Ramanan, Coordinators; Adam Mecca, Study Physician; Oliver Lu, Data System Operator.

\section{Funding}

ADMET 2 is funded by NIA, NIH, R01AG046543. The Sponsor had no role in the design of the trial, and is not involved in data collection, analysis, or interpretation of data.

\section{Availability of data and materials}

Not applicable; no data are presented in this manuscript.

\section{Authors' contributions}

RWS participated in the design and coordination of the study and drafted the manuscript. LTD participated in the design and coordination of the study and critically reviewed and approved the manuscript. KL participated in the conception and design of the study, acquired data, and critically reviewed and approved the manuscript. $\mathrm{NH}$ participated in the conception and design of the study, acquired data, and critically reviewed and approved the manuscript. JM conceived the study, participated in the design of the study, and critically reviewed and approved the manuscript. PP acquired data and critically reviewed and approved the manuscript. OB-M acquired study data and critically reviewed and approved the manuscript. WB acquired data and critically reviewed and approved the manuscript. SC acquired data and critically reviewed and approved the manuscript. AlL acquired data and critically reviewed and approved the manuscript. AL acquired data and critically reviewed and approved the manuscript. CHvD acquired data and critically reviewed and approved the manuscript. All authors read and approved the final manuscript.

\section{Ethics approval and consent to participate}

The institutional review board of the Johns Hopkins Bloomberg School of Public Health reviewed and approved the initial protocol on 9 July 2015, IRB nmber 00006303. Individual clinical centers received approval from their respective IRBs as follows: University of Arkansas, 7 April 2015, protocol 203507; Central Arkansas Veterans Healthcare System institutional review board, 17 August 2015, protocol 639677-3; Western Institutional Review Board, 16 August 2015, protocol 20150480; University Hospitals Case Medical Center institutional review board, 26 May 2015, protocol 05-15-11; Emory University institutional review board, 28 May 2015, IRB00080186; Johns Hopkins Medicine institutional review board, 6 April 2015; IRB00064958; University of Rochester institutional review board, 23 August 2015, RSRB 56395; Roper St. Francis institutional review board, 06 May 2015, protocol 736159-1; Sunnybrook Health Sciences Centre research ethics board, 24 September 2015, 505-2014; Wake Forest University Health Sciences institutional review board, 1 May 2015, IRB 31928; Yale university institutional review board, 10 June 2015, protocol 1503015481. These institutional review boards approved all subsequent amendments to the ADMET 2 protocol. ADMET 2 is being conducted in accordance with the Declaration of Helsinki. Informed consent from both the study participant and his or her caregiver is required prior to study entry and administration of any intervention. Copies of the informed consent template are attached as an appendix.

Consent for publication

Not applicable 


\section{Competing interests}

The authors declare that they have no competing interests.

\section{Publisher's Note}

Springer Nature remains neutral with regard to jurisdictional claims in published maps and institutional affiliations.

\section{Author details}

${ }^{1}$ Johns Hopkins University Bloomberg School of Public Health, Baltimore, MD, USA. ${ }^{2}$ Medical University of South Carolina and Ralph H. Johnson Veterans Administration Medical Center, Charleston, SC, USA. ${ }^{3}$ Sunnybrook Research Institute, Toronto, ON, Canada. ${ }^{4}$ Johns Hopkins University School of Medicine, Baltimore, MD, USA. ${ }^{5}$ University of Arkansas for Medical Science, Central Arkansas Veterans Healthcare System, Little Rock, AR, USA. ${ }^{6}$ Banner Alzheimer's Institute, Phoenix, AZ, USA. 'Wake Forest University School of Medicine, Winston-Salem, NC, USA. ${ }^{8}$ University Hospitals - Case Western Reserve University, Cleveland, OH, USA. ${ }^{9}$ Emory University, Atlanta, GA, USA.

${ }^{10}$ University of Rochester, Rochester, NY, USA. ${ }^{11}$ Yale University, New Haven, $C T$, USA.

\section{Received: 8 June 2017 Accepted: 7 December 2017}

\section{Published online: 18 January 2018}

\section{References}

1. Ferri CP, Prince M, Brayne C, Brodaty H, Fratiglioni L, Ganguli M, et al. Global prevalence of dementia: a Delphi consensus study. Lancet (London, England). 2005;366(9503):2112-7. Epub 2005/12/20. https://doi.org/10.1016/ s0140-6736(05)67889-0. PubMed PMID: 16360788; PubMed Central PMCID: PMCPMC2850264.

2. Rabins PV, Lyketsos CG, Steele CD. Pratical dementia care. 2nd ed. New York: Oxford University Press, Inc:; 2006.

3. Lyketsos CG. Neuropsychiatric symptoms (behavioral and psychological symptoms of dementia) and the development of dementia treatments. Int Psychogeriatr. 1997;19(3):409-20. https://doi.org/10.1017/ S104161020700484X

4. Brodaty $H$, Altendorf A, Withall A, Sachdev P. Do people become more apathetic as they grow older? A longitudinal study in healthy individuals. Int Psychogeriatr. 2010;22(3):426-36. https://doi.org/10.1017/ s1041610209991335. Epub 2009/12/17.

5. Benoit M, Berrut G, Doussaint J, Bakchine S, Bonin-Guillaume S, Fremont $P$, et al. Apathy and depression in mild Alzheimer's disease: a cross-sectional study using diagnostic criteria. J Alzheimers Dis. 2012;31(2):325-34. https:// doi.org/10.3233/jad-2012-112003. Epub 2012/05/01.

6. Benoit M, Robert P. Depression and apathy in Alzheimer's disease. Presse Med (Paris, France: 1983). 2003;32(24 Suppl):S14-8. Epub 2003/09/02.

7. Robert P, Onyike CU, Leentjens AF, Dujardin K, Aalten P, Starkstein S, et al. Proposed diagnostic criteria for apathy in Alzheimer's disease and other neuropsychiatric disorders. Eur Psychiatry. 2009;24(2):98-104. https://doi.org/ 10.1016/j.eurpsy.2008.09.001. Epub 2009/02/10

8. Steinberg M, Shao H, Zandi P, Lyketsos CG, Welsh-Bohmer KA, Norton MC, et al. Point and 5-year period prevalence of neuropsychiatric symptoms in dementia: the Cache County Study. Int J Geriatr Psychiatry. 2008;23(2):170-7. https://doi.org/10.1002/gps.1858. PubMed PMID: 17607801; PubMed Central PMCID: PMCPMC2932652. Epub 2007/07/04.

9. Campbell JJ, Duffy JD. Treatment strategies in amotivated patients. Psychiatr Ann. 1997;27(1):44-9.

10. Greene JG, Smith R, Gardiner M, Timbury GC. Measuring behavioural disturbance of elderly demented patients in the community and its effects on relatives: a factor analytic study. Age Ageing. 1982;11(2):121-6. Epub 1982/05/01.

11. Kaufer DI, Cummings JL, Christine D, Bray T, Castellon S, Masterman D, et al. Assessing the impact of neuropsychiatric symptoms in Alzheimer's disease: the Neuropsychiatric Inventory Caregiver Distress Scale. J Am Geriatr Soc. 1998;46(2):210-5. Epub 1998/02/25

12. Smyth $K$, Neundorfer M, Stuckey J. Progression of Alzheimer's disease, caregiver quality of life, and resource use. Jerusalem, Israel: Eighth Congress of the International Psychogeriatric Association; 1997.

13. Herrmann N, Rothenburg LS, Black SE, Ryan M, Liu BA, Busto UE, et al. Methylphenidate for the treatment of apathy in Alzheimer disease: prediction of response using dextroamphetamine challenge. J Clin Psychopharmacol. 2008;28(3):296-301. https://doi.org/10.1097/JCP. Ob013e318172b479. Epub 2008/05/16.
14. Storga D, Vrecko K, Birkmayer JG, Reibnegger G. Monoaminergic neurotransmitters, their precursors and metabolites in brains of Alzheimer patients. Neurosci Lett. 1996;203(1):29-32. Epub 1996/01/12.

15. van der Linde RM, Dening T, Stephan BC, Prina AM, Evans E, Brayne C. Longitudinal course of behavioural and psychological symptoms of dementia: systematic review. Br J Psychiatry. 2016;209(5):366-77. https://doi. org/10.1192/bjp.bp.114.148403. PubMed PMID: 27491532; PubMed Central PMCID: PMCPMC5100633. Epub 2016/11/03.

16. Salamone JD, Correa M. The mysterious motivational functions of mesolimbic dopamine. Neuron. 2012:76(3):470-85. Epub 2012/11/13. https://doi.org/10.1016/j.neuron.2012.10.021. PubMed PMID: 23141060; PubMed Central PMCID: PMCPMC4450094.

17. Berridge CW, Arnsten AF. Psychostimulants and motivated behavior: arousal and cognition. Neurosci Biobehav Rev. 2013;37(9 Pt A):1976-84. Epub 2012/ 11/21. https://doi.org/10.1016/j.neubiorev.2012.11.005.

18. Roccaforte WH, Burke WJ. Use of psychostimulants for the elderly. Hosp Community Psychiatry. 1990:41(12):1330-3. Epub 1990/12/01.

19. Galynker I, leronimo C, Miner C, Rosenblum J, Vilkas N, Rosenthal R. Methylphenidate treatment of negative symptoms in patients with dementia. J Neuropsychiatry Clin Neurosci. 1997;9(2):231-9. https://doi.org/ 10.1176/jnp.9.2.231. Epub 1997/04/01.

20. Van Reekum R, Bayley M, Garner S, Burke IM, Fawcett S, Hart A, et al. N of 1 study: amantadine for the amotivational syndrome in a patient with traumatic brain injury. Brain Inj. 1995;9(1):49-53. Epub 1995/01/01.

21. Corcoran C, Wong ML, O'Keane V. Bupropion in the management of apathy. J Psychopharmacol. 2004;18(1):133-5. https://doi.org/10.1177/ 0269881104043284. Epub 2004/04/27.

22. Epstein T, Patsopoulos NA, Weiser M. Immediate-release methylphenidate for attention deficit hyperactivity disorder (ADHD) in adults. Cochrane Database Syst Rev. 2014;(9):Cd005041. https://doi.org/10.1002/14651858. CD005041.pub2. Epub 2014/09/19.

23. Marshall GA, Fairbanks LA, Tekin S, Vinters HV, Cummings JL. Neuropathologic correlates of activities of daily living in Alzheimer disease. Alzheimer Dis Assoc Disord. 2006;20(1):56-9. https://doi.org/10.1097/01.wad. 0000201852.60330.16 Epub 2006/02/24.

24. Padala PR, Petty F, Bhatia SC. Methylphenidate may treat apathy independent of depression. Ann Pharmacother. 2005;39(11):1947-9. https:// doi.org/10.1345/aph.1G352. Epub 2005/10/06.

25. Chatterjee A, Fahn S. Methylphenidate treats apathy in Parkinson's disease. J Neuropsychiatry Clin Neurosci. 2002;14(4):461-2. https://doi.org/10.1176/jnp. 14.4.461. Epub 2002/11/12

26. Kittur S, Hauser P. Improvement of sleep and behavior by methylphenidate in Alzheimer's disease. Am J Psychiatry. 1999;156(7):1116-7. https://ajp. psychiatryonline.org/doi/abs/10.1176/ajp.156.7.1116. Epub 1999/07/13.

27. Herrmann N, Lanctot KL, Sambrook R, Lesnikova N, Hebert R, McCracken P, et al. The contribution of neuropsychiatric symptoms to the cost of dementia care. Int J Geriatr Psychiatry. 2006;21(10):972-6. https://doi.org/10. 1002/gps.1594. Epub 2006/09/07.

28. Lanctot KL, Herrmann N, Black SE, Ryan M, Rothenburg LS, Liu BA, et al. Apathy associated with Alzheimer disease: use of dextroamphetamine challenge. Am J Geriatr Psychiatry. 2008;16(7):551-7. https://doi.org/10.1097/ JGP.0b013e318170a6d1. Epub 2008/07/02.

29. Lanctot KL, Moosa S, Herrmann N, Leibovitch FS, Rothenburg L, Cotter A, et al. A SPECT study of apathy in Alzheimer's disease. Dement Geriatr Cogn Disord. 2007:24(1):65-72. https://doi.org/10.1159/000103633. Epub 2007/06/15.

30. Drye LT, Scherer RW, Lanctot KL, Rosenberg PB, Herrmann N, Bachman $D$, et al. Designing a trial to evaluate potential treatments for apathy in dementia: the apathy in dementia methylphenidate trial (ADMET). Am J Geriatr Psychiatry. 2013;21(6):549-59. https://doi.org/10.1016/j.jagp.2012. 12.018, https://doi.org/10.1097/JGP.0b013e31824afba8. PubMed PMID: 23567407; PubMed Central PMCID: PMCPMC3402588. Epub 2013/04/10.

31. Rosenberg PB, Lanctot KL, Drye LT, Herrmann N, Scherer RW, Bachman $\mathrm{DL}$, et al. Safety and efficacy of methylphenidate for apathy in Alzheimer's disease: a randomized, placebo-controlled trial. J Clin Psychiatry. 2013;74(8):810-6. https://doi.org/10.4088/JCP.12m08099. PubMed PMID: 24021498; PubMed Central PMCID: PMCPMC3902018. Epub 2013/09/12

32. Martin BK, Frangakis CE, Rosenberg PB, Mintzer JE, Katz IR, Porsteinsson AP, et al. Design of depression in Alzheimer's disease study-2. Am J Geriatr Psychiatry. 2006:14(11):920-30. https://doi.org/10.1097/01.JGP.0000240977. 71305.ee Epub 2006/10/28. 
33. Schneider LS, Olin JT, Doody RS, Clark CM, Morris JC, Reisberg B, et al. Validity and reliability of the Alzheimer's disease cooperative study-clinical global impression of change. The Alzheimer's disease cooperative study. Alzheimer Dis Assoc Disord. 1997;11 Suppl 2:S22-32. Epub 1997/01/01.

34. Mace NL, Rabins PV. The 36-hour day: a family guide to caring for people who have Alzheimer disease, related dementias, and memory loss. 5th ed. Baltimore, Maryland: The John Hopkins University Press; 2011.

35. Cummings JL. The Neuropsychiatric Inventory: assessing psychopathology in dementia patients. Neurology. 1997;48(5 Suppl 6):S10-6. Epub 1997/05/01.

36. Marin RS, Biedrzycki RC, Firinciogullari S. Reliability and validity of the Apathy Evaluation Scale. Psychiatry Res. 1991;38(2):143-62. Epub 1991/08/01.

37. Strauss ME, Sperry SD. An informant-based assessment of apathy in Alzheimer disease. Neuropsychiatry Neuropsychol Behav Neurol. 2002;15(3): 176-83. Epub 2002/09/10.

38. Galasko D, Bennett D, Sano M, Ernesto C, Thomas R, Grundman M, et al. An inventory to assess activities of daily living for clinical trials in Alzheimer's disease. The Alzheimer's Disease Cooperative Study. Alzheimer Dis Assoc Disord. 1997;11 Suppl 2:S33-9. Epub 1997/01/01.

39. Stern Y, Albert SM, Sano M, Richards M, Miller L, Folstein M, et al. Assessing patient dependence in Alzheimer's disease. J Gerontol. 1994;49(5):M216-22. Epub 1994/09/01.

40. Johnson JA, Coons SJ, Ergo A, Szava-Kovats G. Valuation of EuroQOL (EQ-5D) health states in an adult US sample. Pharmacoeconomics. 1998; 13(4):421-33. Epub 1998/03/08.

41. Wimo A, Gustavsson A, Jonsson L, Winblad B, Hsu MA, Gannon B. Application of Resource Utilization in Dementia (RUD) instrument in a global setting. Alzheimers Dement. 2013;9(4):429-35.e17. https://doi.org/10. 1016/j.jalz.2012.06.008. Epub 2012/11/13.

42. Folstein MF, Folstein SE, McHugh PR. "Mini-mental state". A practical method for grading the cognitive state of patients for the clinician. J Psychiatr Res. 1975;12(3):189-98. Epub 1975/11/01.

43. Brandt J, Benedict RHB. Hopkins verbal learning test-revised professional manual: Psychological Assessment Resources. 2001.

44. Wechsler D. Wechsler adult intelligence scale-revised manual. New York: The Psychological Corporation; 1981.

45. Stuss DT, Bisschop SM, Alexander MP, Levine B, Katz D, Izukawa D. The Trail Making Test: a study in focal lesion patients. Psychol Assess. 2001;13(2):230-9. Epub 2001/07/04.

46. Piatt AL, Fields JA, Paolo AM, Troster Al. Action (verb naming) fluency as an executive function measure: convergent and divergent evidence of validity. Neuropsychologia. 1999;37(13):1499-503. Epub 2000/01/05.

47. Isaacs B, Akhtar AJ. The set test: a rapid test of mental function in old people. Age Ageing. 1972;1(4):222-6. Epub 1972/11/01.

48. Franzen MD, Haut MW, Rankin E, Keefover R. Empriical comparison of alternate forms of the Boston Naming Test. Clin Neuropsychol. 1995;9(3): 225-9. http://dx.doi.org/10.1080/13854049508400484.

49. Whitehead J. Sample size calculations for ordered categorical data. Stat Med. 1993;12(24):2257-71. Epub 1993/12/30.

\section{Submit your next manuscript to BioMed Central and we will help you at every step:}

- We accept pre-submission inquiries

- Our selector tool helps you to find the most relevant journal

- We provide round the clock customer support

- Convenient online submission

- Thorough peer review

- Inclusion in PubMed and all major indexing services

- Maximum visibility for your research

Submit your manuscript at www.biomedcentral.com/submit 